\title{
Some modular considerations regarding odd perfect numbers
}

\author{
Jose Arnaldo Bebita Dris ${ }^{1}$ and Immanuel Tobias San Diego ${ }^{2}$ \\ ${ }^{1}$ M. Sc. Graduate, Mathematics Department \\ De La Salle University, Manila, Philippines 1004 \\ e-mail: josearnaldobdris@gmail.com \\ ${ }^{2}$ Department of Mathematics and Physical Sciences \\ Trinity University of Asia, Quezon City, Philippines 1102 \\ e-mail: itsandiego@tua.edu.ph
}

Received: 7 April 2019

Revised: 6 January 2020

Accepted: 20 April 2020

\begin{abstract}
Let $p^{k} m^{2}$ be an odd perfect number with special prime $p$. In this article, we provide an alternative proof for the biconditional that $\sigma\left(m^{2}\right) \equiv 1(\bmod 4)$ holds if and only if $p \equiv k(\bmod 8)$. We then give an application of this result to the case when $\sigma\left(m^{2}\right) / p^{k}$ is a square.
\end{abstract}

Keywords: Sum of divisors, Sum of aliquot divisors, Deficiency, Odd perfect number, Special prime.

2010 Mathematics Subject Classification: 11A05, 11A25.

\section{Introduction}

Let $\sigma(z)$ denote the sum of the divisors of $z \in \mathbb{N}$, the set of positive integers. Denote the deficiency [5] of $z$ by $D(z)=2 z-\sigma(z)$, and the sum of the aliquot divisors [6] of $z$ by $s(z)=\sigma(z)-z$. Note that we have the identity $D(z)+s(z)=z$.

If $n$ is odd and $\sigma(n)=2 n$, then $n$ is said to be an odd perfect number [8]. Euler proved that an odd perfect number, if one exists, must have the form $n=p^{k} m^{2}$, where $p$ is the special prime satisfying $p \equiv k \equiv 1(\bmod 4)$ and $\operatorname{gcd}(p, m)=1$. 
Chen and Luo [2] gave a characterization of the forms of odd perfect numbers $n=p^{k} m^{2}$ such that $p \equiv k(\bmod 8)$. Starni [7] proved that there is no odd perfect number decomposable into primes all of the type $\equiv 1(\bmod 4)$ if $n=p^{k} m^{2}$ and $p \not \equiv k(\bmod 8)$. Starni used a congruence from Ewell [3] to prove this result.

Note that, in general, since $m^{2}$ is a square, we get

$$
\sigma\left(m^{2}\right) \equiv 1 \quad(\bmod 2) \text {. }
$$

This paper provides an alternative proof for Theorem 3.3, equation 3.1 in Chen and Luo's article titled "Odd multiperfect numbers" [2]:

Theorem 1.1. Let $n=\pi^{\alpha} M^{2}$ be an odd 2-perfect number, with $\pi$ prime, $\operatorname{gcd}(\pi, M)=1$ and $\pi \equiv \alpha \equiv 1(\bmod 4)$. Then

$$
\sigma\left(M^{2}\right) \equiv 1 \quad(\bmod 4) \Longleftrightarrow \pi \equiv \alpha \quad(\bmod 8)
$$

The method presented in this paper may potentially be used to extend the arguments to consider $\sigma\left(m^{2}\right)$ modulo 8 .

\section{Preliminaries}

Starting from the fundamental equality

$$
\frac{\sigma\left(m^{2}\right)}{p^{k}}=\frac{2 m^{2}}{\sigma\left(p^{k}\right)}
$$

(which follows from the facts that $\sigma(n)=2 n, \sigma$ is multiplicative, and $\operatorname{gcd}\left(p^{k}, \sigma\left(p^{k}\right)\right)=1$ ), one can derive

$$
\frac{\sigma\left(m^{2}\right)}{p^{k}}=\frac{2 m^{2}}{\sigma\left(p^{k}\right)}=\operatorname{gcd}\left(m^{2}, \sigma\left(m^{2}\right)\right)
$$

so that we ultimately have

$$
\frac{D\left(m^{2}\right)}{s\left(p^{k}\right)}=\frac{2 m^{2}-\sigma\left(m^{2}\right)}{\sigma\left(p^{k}\right)-p^{k}}=\operatorname{gcd}\left(m^{2}, \sigma\left(m^{2}\right)\right)
$$

and

whereby we obtain

$$
\frac{s\left(m^{2}\right)}{D\left(p^{k}\right) / 2}=\frac{\sigma\left(m^{2}\right)-m^{2}}{p^{k}-\frac{\sigma\left(p^{k}\right)}{2}}=\operatorname{gcd}\left(m^{2}, \sigma\left(m^{2}\right)\right),
$$

$$
\frac{D\left(p^{k}\right) D\left(m^{2}\right)}{s\left(p^{k}\right) s\left(m^{2}\right)}=2 .
$$

Note that we also have the following equation:

$$
\frac{2 D\left(m^{2}\right) s\left(m^{2}\right)}{D\left(p^{k}\right) s\left(p^{k}\right)}=\left(\operatorname{gcd}\left(m^{2}, \sigma\left(m^{2}\right)\right)\right)^{2} .
$$

Lastly, notice that we can easily get

$$
\sigma\left(p^{k}\right) \equiv k+1 \equiv 2 \quad(\bmod 4)
$$

(since $p \equiv k \equiv 1(\bmod 4))$ so that it remains to consider the possible equivalence classes for $\sigma\left(m^{2}\right)$ modulo 4 . Since $\sigma\left(m^{2}\right)$ is odd, we only need to consider two.

We ask: Which equivalence class of $\sigma\left(m^{2}\right)$ modulo 4 makes Equation $(*)$ untenable? 


\section{Discussion and results}

We know that the answer to the question we posed in the previous section must somehow depend on the equivalence class of $p$ and $k$ modulo 8 , but as we only know that $p \equiv k \equiv 1(\bmod 4)$, we need to consider the following cases separately and thereby prove the corresponding results:

Remark 3.1. Suppose that $n=p^{k} m^{2}$ is an odd perfect number with special prime $p$. We claim the truth of the following propositions, which we will need to treat separately later:

1. If $p \equiv k \equiv 1(\bmod 8)$, then $\sigma\left(m^{2}\right) \equiv 3(\bmod 4)$ is impossible.

2. If $p \equiv 1(\bmod 8)$ and $k \equiv 5(\bmod 8)$, then $\sigma\left(m^{2}\right) \equiv 1(\bmod 4)$ is impossible.

3. If $p \equiv 5(\bmod 8)$ and $k \equiv 1(\bmod 8)$, then $\sigma\left(m^{2}\right) \equiv 1(\bmod 4)$ is impossible.

4. If $p \equiv k \equiv 5(\bmod 8)$, then $\sigma\left(m^{2}\right) \equiv 3(\bmod 4)$ is impossible.

First, we prove the following lemmas:

Lemma 3.2. Suppose that $n=p^{k} m^{2}$ is an odd perfect number with special prime $p$.

1. If $p \equiv 1(\bmod 8)$, then $\sigma\left(p^{k}\right) \equiv k+1(\bmod 8)$.

2. If $p \equiv 5(\bmod 8)$ and $k \equiv 1(\bmod 8)$, then $\sigma\left(p^{k}\right) \equiv 6(\bmod 8)$.

3. If $p \equiv 5(\bmod 8)$ and $k \equiv 5(\bmod 8)$, then $\sigma\left(p^{k}\right) \equiv 2(\bmod 8)$.

Proof. Let $n=p^{k} m^{2}$ be an odd perfect number with special prime $p$. It follows that $p \equiv 1$ $(\bmod 4)$.

We consider two cases:

Case 1: $p \equiv 1(\bmod 8)$ We obtain

$$
\sigma\left(p^{k}\right)=\sum_{i=0}^{k} p^{i} \equiv 1+\sum_{i=1}^{k} p^{i} \equiv 1+\sum_{i=1}^{k} 1^{i} \equiv k+1 \quad(\bmod 8)
$$

as desired.

Case $2: p \equiv 5(\bmod 8)$ We get

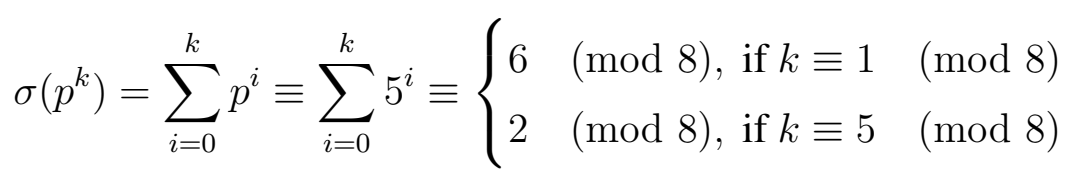

This completes the proof.

Lemma 3.3. Suppose that $n=p^{k} m^{2}$ is an odd perfect number with special prime $p$.

1. If $p \equiv 1(\bmod 8)$ and $k \equiv 1(\bmod 8)$, then $D\left(p^{k}\right) \equiv 0(\bmod 8)$.

2. If $p \equiv 1(\bmod 8)$ and $k \equiv 5(\bmod 8)$, then $D\left(p^{k}\right) \equiv 4(\bmod 8)$.

3. If $p \equiv 5(\bmod 8)$ and $k \equiv 1(\bmod 8)$, then $D\left(p^{k}\right) \equiv 4(\bmod 8)$. 
4. If $p \equiv 5(\bmod 8)$ and $k \equiv 5(\bmod 8)$, then $D\left(p^{k}\right) \equiv 0(\bmod 8)$.

Proof. The proof is trivial and follows directly from Lemma 3.2, using the formula $D\left(p^{k}\right)=2 p^{k}-\sigma\left(p^{k}\right)$.

Lemma 3.4. Suppose that $n=p^{k} m^{2}$ is an odd perfect number with special prime $p$.

1. If $p \equiv 1(\bmod 8)$ and $k \equiv 1(\bmod 8)$, then $s\left(p^{k}\right) \equiv 1(\bmod 8)$.

2. If $p \equiv 1(\bmod 8)$ and $k \equiv 5(\bmod 8)$, then $s\left(p^{k}\right) \equiv 5(\bmod 8)$.

3. If $p \equiv 5(\bmod 8)$ and $k \equiv 1(\bmod 8)$, then $s\left(p^{k}\right) \equiv 1(\bmod 8)$.

4. If $p \equiv 5(\bmod 8)$ and $k \equiv 5(\bmod 8)$, then $s\left(p^{k}\right) \equiv 5(\bmod 8)$.

Proof. The proof is trivial and follows directly from Lemma 3.3, using the formula $s\left(p^{k}\right)=p^{k}-D\left(p^{k}\right)$.

Lemma 3.5. Suppose that $n=p^{k} m^{2}$ is an odd perfect number with special prime $p$.

1. If $\sigma\left(m^{2}\right) \equiv 1(\bmod 4)$, then $D\left(m^{2}\right) \equiv 1(\bmod 4)$.

2. If $\sigma\left(m^{2}\right) \equiv 3(\bmod 4)$, then $D\left(m^{2}\right) \equiv 3(\bmod 4)$.

Proof. The proof is trivial and follows directly from the fact that $m^{2} \equiv 1(\bmod 4)$ (since $m$ is odd), using the underlying assumptions and the formula $D\left(m^{2}\right)=2 m^{2}-\sigma\left(m^{2}\right)$.

Lemma 3.6. Suppose that $n=p^{k} m^{2}$ is an odd perfect number with special prime $p$.

1. If $\sigma\left(m^{2}\right) \equiv 1(\bmod 4)$, then $s\left(m^{2}\right) \equiv 0(\bmod 4)$.

2. If $\sigma\left(m^{2}\right) \equiv 3(\bmod 4)$, then $s\left(m^{2}\right) \equiv 2(\bmod 4)$.

Proof. The proof is trivial and follows directly from Lemma 3.5, using the formula $s\left(m^{2}\right)=m^{2}-D\left(m^{2}\right)$.

We are now ready to prove our main result.

Theorem 3.7. Suppose that $n=p^{k} m^{2}$ is an odd perfect number with special prime $p$.

1. If $p \equiv k \equiv 1(\bmod 8)$, then $\sigma\left(m^{2}\right) \equiv 3(\bmod 4)$ is impossible.

2. If $p \equiv 1(\bmod 8)$ and $k \equiv 5(\bmod 8)$, then $\sigma\left(m^{2}\right) \equiv 1(\bmod 4)$ is impossible.

3. If $p \equiv 5(\bmod 8)$ and $k \equiv 1(\bmod 8)$, then $\sigma\left(m^{2}\right) \equiv 1(\bmod 4)$ is impossible.

4. If $p \equiv k \equiv 5(\bmod 8)$, then $\sigma\left(m^{2}\right) \equiv 3(\bmod 4)$ is impossible. 
Proof. Let $n=p^{k} m^{2}$ be an odd perfect number with special prime $p$.

Notice that the right-hand side of Equation $(*)$ is odd. (Furthermore, it is congruent to 1 modulo 8.)

First, suppose that $p \equiv k \equiv 1(\bmod 8)$, and assume to the contrary that $\sigma\left(m^{2}\right) \equiv 3(\bmod 4)$ holds. By Lemma 3.3, $D\left(p^{k}\right) \equiv 0(\bmod 8)$. By Lemma 3.5, $D\left(m^{2}\right) \equiv 3(\bmod 4)$. By Lemma 3.4, $s\left(p^{k}\right) \equiv 1(\bmod 8)$. By Lemma 3.6, $s\left(m^{2}\right) \equiv 2(\bmod 4)$. Thus, from Equation $(*)$ we obtain (symbolically)

$$
2\left(4 a_{1}+3\right)\left(4 b_{1}+2\right)=\left(8 x_{1}+1\right)\left(8 c_{1}\right)\left(8 d_{1}+1\right),
$$

which does not have any integer solutions.

Next, suppose that $p \equiv 1(\bmod 8)$ and $k \equiv 5(\bmod 8)$, and assume to the contrary that $\sigma\left(m^{2}\right) \equiv 1(\bmod 4)$ holds. By Lemma 3.3, $D\left(p^{k}\right) \equiv 4(\bmod 8)$. By Lemma 3.5, $D\left(m^{2}\right) \equiv 1$ $(\bmod 4)$. By Lemma 3.4, $s\left(p^{k}\right) \equiv 5(\bmod 8)$. By Lemma 3.6, $s\left(m^{2}\right) \equiv 0(\bmod 4)$. Thus, from Equation $(*)$ we obtain (symbolically)

$$
2\left(4 a_{2}+1\right)\left(4 b_{2}\right)=\left(8 x_{2}+1\right)\left(8 c_{2}+4\right)\left(8 d_{2}+5\right),
$$

which does not have any integer solutions.

Now, suppose that $p \equiv 5(\bmod 8)$ and $k \equiv 1(\bmod 8)$, and assume to the contrary that $\sigma\left(m^{2}\right) \equiv 1(\bmod 4)$ holds. By Lemma 3.3, $D\left(p^{k}\right) \equiv 4(\bmod 8)$. By Lemma 3.5, $D\left(m^{2}\right) \equiv 1$ $(\bmod 4)$. By Lemma 3.4, $s\left(p^{k}\right) \equiv 1(\bmod 8)$. By Lemma 3.6, $s\left(m^{2}\right) \equiv 0(\bmod 4)$. Thus, from Equation $(*)$ we obtain (symbolically)

$$
2\left(4 a_{3}+1\right)\left(4 b_{3}\right)=\left(8 x_{3}+1\right)\left(8 c_{3}+4\right)\left(8 d_{3}+1\right),
$$

which does not have any integer solutions.

Finally, suppose that $p \equiv k \equiv 5(\bmod 8)$, and assume to the contrary that $\sigma\left(m^{2}\right) \equiv 3$ $(\bmod 4)$ holds. By Lemma 3.3, $D\left(p^{k}\right) \equiv 0(\bmod 8)$. By Lemma 3.5, $D\left(m^{2}\right) \equiv 3(\bmod 4)$. By Lemma $3.4, s\left(p^{k}\right) \equiv 5(\bmod 8)$. By Lemma 3.6, $s\left(m^{2}\right) \equiv 2(\bmod 4)$. Thus, from Equation $(*)$ we obtain (symbolically)

$$
2\left(4 a_{4}+3\right)\left(4 b_{4}+2\right)=\left(8 x_{4}+1\right)\left(8 c_{4}\right)\left(8 d_{4}+5\right),
$$

which does not have any integer solutions.

This concludes the proof.

Remark 3.8. To summarize, Theorem 3.7 just states that if $n=p^{k} m^{2}$ is an odd perfect number with a special prime $p$, then $\sigma\left(m^{2}\right) \equiv 1(\bmod 4)$ holds if and only if $p \equiv k(\bmod 8)$. Our argument provides an alternative proof for Theorem 3.3, equation 3.1 in [2] (as reproduced above in Theorem 1.1).

\section{An application}

Let $n=p^{k} m^{2}$ be an odd perfect number with special prime $p$, and let $\sigma\left(m^{2}\right) / p^{k}$ be a square. Since $\sigma\left(m^{2}\right) / p^{k}$ is odd, it follows that $\sigma\left(m^{2}\right) / p^{k} \equiv 1(\bmod 4)$. But it is known that $p \equiv k \equiv 1$ 
$(\bmod 4)$. In particular, we know that $p^{k} \equiv 1(\bmod 4)$. This implies that $\sigma\left(m^{2}\right) \equiv 1(\bmod 4)$, if $\sigma\left(m^{2}\right) / p^{k}$ is a square. By Theorem 3.7, we know that $p \equiv k(\bmod 8)$.

Moreover, Broughan, Delbourgo, and Zhou proved in [1] (Lemma 8, page 7) that if $\sigma\left(m^{2}\right) / p^{k}$ is a square, then $k=1$ holds.

Thus, under the assumption that $\sigma\left(m^{2}\right) / p^{k}$ is a square, we have

$$
p \equiv k=1 \quad(\bmod 8) \text {. }
$$

This implies that the lowest possible value for the special prime $p$ is 17 .

We state this result as our next theorem.

Theorem 4.1. Suppose that $n=p^{k} m^{2}$ is an odd perfect number with special prime $p$. If $\sigma\left(m^{2}\right) / p^{k}$ is a square, then $p \geq 17$.

Remark 4.2. Let $n=p^{k} m^{2}$ be an odd perfect number with special prime $p$.

Note that if

$$
\frac{\sigma\left(m^{2}\right)}{p^{k}}=\frac{m^{2}}{\sigma\left(p^{k}\right) / 2}
$$

is a square, then $k=1$ and $\sigma\left(p^{k}\right) / 2=(p+1) / 2$ is also a square.

The possible values for the special prime satisfying $p<100$ and $p \equiv 1(\bmod 8)$ are 17,41 , 73, 89, and 97.

For each of these values:

$$
\begin{aligned}
& \frac{p_{1}+1}{2}=\frac{17+1}{2}=9=3^{2} . \\
& \frac{p_{2}+1}{2}=\frac{41+1}{2}=21, \text { which is not a square. } \\
& \frac{p_{3}+1}{2}=\frac{73+1}{2}=37, \text { which is not a square. } \\
& \frac{p_{4}+1}{2}=\frac{89+1}{2}=45, \text { which is not a square. } \\
& \frac{p_{5}+1}{2}=\frac{97+1}{2}=49=7^{2} .
\end{aligned}
$$

A quick way to rule out 41, 73 and 89, as remarked by Ochem [4] over at Mathematics StackExchange, is as follows: "If $(p+1) / 2$ is an odd square, then $(p+1) / 2 \equiv 1(\bmod 8)$, so that $p \equiv 1(\bmod 16)$. This rules out 41,73 , and $89 . "$

\section{Conclusion}

Additional tools are required if we are to push the analysis from $\sigma\left(m^{2}\right)$ modulo 4 to consider $\sigma\left(m^{2}\right)$ modulo 8 . The authors have tried to check Equation $(*)$ by considering $m^{2} \equiv 1(\bmod 8)$, and the various corresponding cases for $\sigma\left(m^{2}\right)$ modulo 8 (which are determined by Theorem 3.7), but so far all their attempts have not resulted in any contradictions. 


\section{Acknowledgements}

The authors are indebted to the anonymous referees whose valuable feedback improved the overall presentation and style of this manuscript.

\section{References}

[1] Broughan, K. A., Delbourgo, D., \& Zhou, Q. (2013). Improving the Chen and Chen result for odd perfect numbers, Integers, 13, Article \#A39.

[2] Chen, S.-C., \& Luo, H. (2013). Odd multiperfect numbers, Bulletin of the Australian Mathematical Society, 88 (1), 56-63.

[3] Ewell, J. A. Jr. (1980). On the multiplicative structure of odd perfect numbers, Journal of Number Theory, 12, 339-342.

[4] Ochem, P. (2019). Answer to a question of the first author in Mathematics StackExchange, https://math.stackexchange.com/a/3151412/28816.

[5] Sloane, N. J. A., OEIS sequence A033879 - Deficiency of $n$, or $2 n-\sigma(n)$, https: // oeis.org/A033879.

[6] Sloane, N. J. A., \& Guy, R. K., OEIS sequence A001065 - Sum of proper divisors (or aliquot parts) of $n$ : sum of divisors of $n$ that are less than $n$, https: / / oeis.org/A001065.

[7] Starni, P. (1991). On the Euler's factor of an odd perfect number, Journal of Number Theory, 37, 366-369.

[8] Wikipedia contributors. (2019, March 6). Perfect number. In Wikipedia, The Free Encyclopedia. Retrieved from https://en.wikipedia.org/w/index.php? title=Perfect_number\&oldid=886493275. 